\section{Cureus}

\title{
An Unusual Presentation of Systemic Lupus Erythematosus as Hemophagocytic Lymphohistiocytosis in a Male.
}

\author{
Mathew Thomas ${ }^{1}$, Alex Robert ${ }^{2}$, Neenu Kuruvilla ${ }^{3}$, Uthamanand $C^{2}$ \\ 1. Breast Medical Oncology, Cleveland Clinic, Cleveland, USA 2. Internal Medicine, Church of South \\ India (CSI) Holdsworth Memorial Hospital, Mysore, IND 3. Internal Medicine, Government Medical \\ College Kottayam, Kottayam, IND
}

$\square$ Corresponding author: Mathew Thomas, thomasm4@ccf.org

Disclosures can be found in Additional Information at the end of the article

\section{Abstract}

Hemophagocytic lymphohistiocytosis (HLH) is an aggressive and life-threatening hyperinflammatory condition characterized by excessive activation of macrophages and $\mathrm{T}$ cells resulting in multi-organ dysfunction. HLH can be primary/familial or secondary to infections, malignancy, immunosuppression, and autoimmune conditions. Systemic lupus erythematosus (SLE) is an autoimmune condition that can predispose to HLH. SLE, as other immune conditions is more common in females than in males. However, the occurrence of SLE in males and subsequent predisposition to HLH is rare. We report the case of a 22-year-old gentleman who presented with fever for three months and one day of altered sensorium prior to admission. On evaluation, he fulfilled five out of the 17 diagnostic criteria for SLE. His bone marrow biopsy showed hemophagocytosis and met five out of the eight diagnostic criteria of HLH, and a diagnosis of HLH secondary to SLE was made. He was treated with pulse doses of intravenous methylprednisolone and azathioprine and showed dramatic improvement. A high index of suspicion is essential for the diagnosis of HLH and prompt initiation of treatment is of utmost importance for tackling such a rapidly progressive life-threatening condition.

Categories: Internal Medicine, Allergy/Immunology, Rheumatology

Keywords: hlh, male sle, autoimmune, rare presentation

\section{Introduction}

Hemophagocytic lymphohistiocytosis (HLH) is an aggressive and potentially fatal condition characterized by immune activation leading to multi-organ dysfunction. HLH can be inherited in an autosomal recessive fashion, but can also be secondary to infections, malignancy, immunosuppression and autoimmune conditions [1]. Primary HLH is more common in the pediatric population, while secondary HLH is more common among adults [1]. HLH involves inappropriate activation of $\mathrm{T}$ cells and macrophages, which produces pro-inflammatory cytokines [1]. Common manifestations of HLH include prolonged fever, hepatosplenomegaly, pancytopenia, and elevated levels of liver enzymes, triglyceride, and ferritin. Systemic lupus erythematosus (SLE) is an autoimmune condition that can predispose to HLH. The occurrence of SLE in males and subsequent predisposition to HLH is rare, with a prevalence of $0.9 \%$ to 4.6 $\%$ [2]. Here, we report a rare case of young male with SLE, whose initial manifestation was HLH.

\section{Case Presentation}

A 22-year-old male, hotel employee by profession, with no significant past medical history and 


\section{Cureus}

family history, presented with fever for three months and one day of altered sensorium. He had intermittent low grade fever for three months, but it got worsened over the five days prior to admission. It was associated with chills and with 1-2 episodes/day of vomiting for three days. He also had a history of leg swelling, facial puffiness, and abdominal distension for three days. There was no history of chest pain, shortness of breath, palpitations, headache, syncope, or seizures. He denied any history of smoking, excessive alcohol use, or substance abuse. On examination, the patient was drowsy and disoriented with a Glasgow Coma Score (GCS) of $12 / 15$, temperature of $101^{\circ} \mathrm{F}$, pulse rate of 96/min, blood pressure of $132 / 84 \mathrm{~mm} \mathrm{Hg}$, and Spo2 of $96 \%$ in room air. Physical examination showed the presence of pallor, facial puffiness, and bilateral pitting pedal edema. Systemic examination showed the presence of hepatosplenomegaly and shifting dullness. There were no signs of meningeal irritation, no focal deficits, and optic fundus examination was normal.

\section{Investigations and treatment}

Laboratory results at presentation (Table 1) were significant for pancytopenia, hyponatremia, hypoalbuminemia, hyperbilirubinemia, and elevated liver enzymes.

\begin{tabular}{|c|c|c|}
\hline Variable & Measurement & Reference values \\
\hline Hemoglobin (g/dL) & 5.8 & 13.5-17.5 \\
\hline Total leucocyte count (TLC) (/mm3) & 1000 & $4,500-11,000$ \\
\hline Neutrophil (\%) & 79 & $54-62$ \\
\hline Lymphocytes (\%) & 12 & $25-33$ \\
\hline Monocytes (\%) & 4 & $3-7$ \\
\hline Eosinophil (\%) & 0.8 & $1-3$ \\
\hline Basophil (\%) & 2.4 & $0-0.75$ \\
\hline Platelet count(/mm3) & 66000 & $150,000-400,000$ \\
\hline MCV $(\mu \mathrm{m} 3)$ & 75 & $80-100$ \\
\hline ESR (mm/h) & 18 & $0-15$ \\
\hline Sodium (mEq/L) & 128 & 136-145 \\
\hline Potassium (mEq/L) & 5.2 & $3.5-5.0$ \\
\hline Chloride (mEq/L) & 99 & 95-105 \\
\hline Blood urea nitrogen (mmol/dL) & 98 & $8-24$ \\
\hline Creatinine (mg/dL) & 1.1 & $0.6-1.2$ \\
\hline Total protein (g/dL) & 4.8 & $6.0-7.8$ \\
\hline Albumin (g/dL) & 1.7 & $3.5-5.5$ \\
\hline Total bilirubin (mg/dL) & 1.4 & $0.1-1.0$ \\
\hline Direct bilirubin (mg/dL) & 1.0 & $0.0-0.3$ \\
\hline
\end{tabular}




\section{Cureus}

$\begin{array}{lll}\text { AST }(U / L) & 159 & 8-40 \\ \text { ALT }(U / L) & 34 & 8-40 \\ \text { ALP }(U / L) & 214 & 30-100 \\ \text { PT (seconds) } & 13.6 & 11-15 \\ \text { INR } & 0.71 & 0.8-1.2 \\ \text { MP-QBC } & \text { Negative } \\ \text { Urine-albumin, sugar } & \text { Nil } & \\ \text { Urine-pus cells } & 1-2 / \mathrm{hpf} & \\ \text { Urine-RBC } & \text { Nil }\end{array}$

\section{TABLE 1: Laboratory results at presentation}

MCV: mean corpuscular volume; ESR: erythrocyte sedimentation rate; AST: aspartate aminotransferase; ALT: alanine aminotransferase; ALP: alkaline phosphatase; PT: prothrombin time; INR: international normalized ratio; MP-QBC: malarial parasitequantitative buffy coat; RBC: red blood cell; hpf: high power field

Computed tomography (CT) of the brain was normal. Ultrasound of abdomen and pelvis showed hepatosplenomegaly with moderate-to-severe ascites. Results of other investigations including peripheral smear, infectious disease panel, and Coombs test are shown in Table 2. The initial differential diagnoses were autoimmune, infectious, or inflammatory conditions. So he was started on empiric doxycycline, meropenem, hydrocortisone, fluconazole, and other supportive measures. 


\section{Cureus}

\begin{tabular}{|c|c|c|}
\hline Variable & Measurement & Reference values \\
\hline Peripheral smear & Microcytic hypochromic RBC, leucopenia, thrombocytopenia, no blasts & \\
\hline HIV ELISA & Negative & \\
\hline HBsAg & Negative & \\
\hline Anti-HCV antibody & Negative & \\
\hline Reticulocyte Count & $1 \%$ & $0.5 \%-1.5 \%$ of red cells \\
\hline Weil-Felix test & Negative & \\
\hline Widal test & Negative & \\
\hline IgM Scrub typhus & Negative & \\
\hline lgM Brucella & Negative & \\
\hline IgM Leptospira & Negative & \\
\hline lgM Dengue & Negative & \\
\hline TSH $(\mu \mathrm{U} / \mathrm{mL})$ & 0.63 & $0.5-5.0$ \\
\hline Direct Coombs test & Negative & \\
\hline Indirect Coombs test & Negative & \\
\hline Blood Culture & No growth (48 hrs) and after 5 days & \\
\hline
\end{tabular}

\section{TABLE 2: Infectious disease panel}

ELISA: enzyme-linked immunosorbent assay; RBC: red blood cell; HBsAg: hepatitis B surface antigen; HCV: hepatitis C virus; IgM: immunoglobulin M; HIV: human immunodeficiency virus; TSH: thyroid-stimulating hormone 


\section{Cureus}

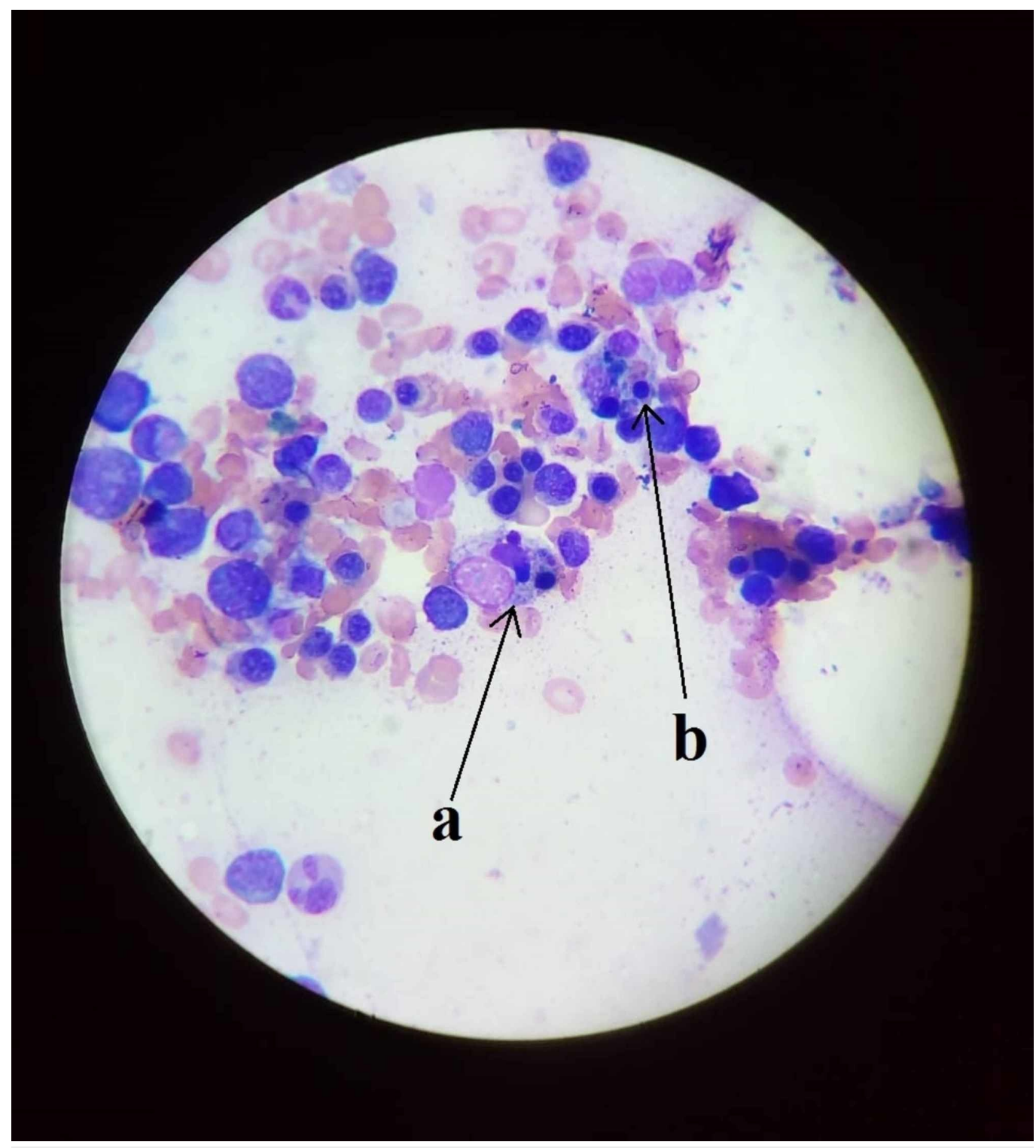

FIGURE 1: Bone marrow biopsy showing hemophagocytosis and lupus erythematosus cell phenomenon

(a) Lupus erythematosus cell, (b) phagocytosis by histiocyte 


\section{Cureus}

\begin{tabular}{|c|c|c|}
\hline Variable & Measurement & Reference values \\
\hline LDH & 2944 & 45-90 U/L (100-250 IU/L) \\
\hline Ferritin (ng/mL) & 1896 & $15-200$ \\
\hline ANA & & Impression \\
\hline Intensity & $3+$ & \multirow{5}{*}{ Positive ANA if there is positive reaction with 1:100 titer } \\
\hline Titer & 1:100 & \\
\hline Pattern & Nuclear homogenous & \\
\hline Impression & positive & \\
\hline Anti-dsDNA & positive & \\
\hline
\end{tabular}

\section{TABLE 3: Autoimmune and hemophagocytic lymphohistiocytosis panel}

LDH: lactate dehydrogenase; ANA: antinuclear antibody; Anti-ds DNA: antidouble stranded DNA

He was then treated with pulse dose steroids with intravenous methylprednisolone $1000 \mathrm{mg}$ daily for five days and then switched to prednisolone $60 \mathrm{mg}$ orally (PO) once daily. With blood transfusions and other supportive measures, his blood counts improved. Later, steroids were tapered and azathioprine $50 \mathrm{mg}$ PO twice daily was added. His general condition improved over the next one week. There were no more episodes of fever and altered sensorium, and he was discharged after three weeks on steroids and immunosuppressants. Follow-up visit at one week showed significant improvement. Now he is on regular outpatient follow up and is on azathioprine PO daily and tapering doses of steroids. He is doing well clinically and back to his routine day to day work.

\section{Discussion}

We report a case of young male patient whose SLE manifested as HLH. His clinical and laboratory findings indicated pancytopenia, positive ANA and anti-ds DNA antibody, meeting five out of 17 (three clinical and two immunological) of the Systemic Lupus International Collaborating Clinics (SLICC) criteria for the diagnosis of SLE [3]. He had fever, pancytopenia, splenomegaly, bone marrow hemophagocytosis, and hyperferritinemia, fulfilling five out of the eight diagnostic criteria of HLH, described in the HLH 2004 trial [4-5]. Hence, the patient was diagnosed with SLE manifesting as HLH.

HLH can be primary resulting from a genetic defect leading to decreased cytotoxic activity of Natural Killer (NK) cells and cytotoxic T cells [2]. Secondary HLH results from triggering agents like infections or malignancies but can also be induced by autoimmune conditions, in which case it is called macrophage activation syndrome (MAS) [1]. Inability to clear the antigens secondary to these conditions lead to hyperactivation of the immune system, progressing to cytokine storm, and results in organ dysfunction [1]. HLH possesses diagnostic challenges as it has overlapping features with sepsis and multiple organ dysfunction syndrome (MODS) [1].

The treatment of HLH is based on the HLH-94 protocol. Induction/initial therapy consists of etoposide (twice weekly during the first two weeks, and then weekly), in combination with 
dexamethasone [6]. Central nervous system (CNS) disease is treated with intrathecal methotrexate. For patients with resistant or relapsing disease, continuation therapy with pulse doses of dexamethasone in combination with etoposide and cyclosporine is recommended to keep them in remission until allogeneic hematopoietic stem cell transplant could be performed.

In 2004, the HLH-2004 protocol was initiated (NCT00426101). It differs from HLH-94 protocol by using cyclosporine from day one of the initial treatment phase, and the addition of intrathecal steroids to intrathecal methotrexate for CNS disease. The HLH-94 and 2004 protocols are pediatric protocols, and may result in overtreatment and toxic when used in adults. Hence, dose reductions and individualized approach should be considered [1].

Antithymocyte globulin is an immunosuppressant effective in familial HLH [7]. Emapalumab is an anti-interferon (IFN)- $\gamma$ monoclonal antibody approved by the Food and Drug

Administration (FDA) in 2018, for the treatment of primary HLH in adults and children with refractory, recurrent, or progressive disease [8]. Alemtuzumab is an anti CD-52 monoclonal antibody, considered as an effective salvage treatment agent for refractory HLH, leading to improvement and survival benefit to hematopoietic stem cell transplant [9].

Treatment of secondary HLH is aimed at treating the underlying condition. In HLH due to SLE, high dose steroids and immunosuppressive agents including cyclosporine, cyclophosphamide, and intravenous immunoglobulin are effective [10]. Aoyama-Maeda et al. reported a case of MAS secondary to SLE, successfully treated with intravenous immunoglobulin (IVIG) and oral tacrolimus [11]. There are reported cases of treatment refractory SLE induced HLH, managed successfully with rituximab and infliximab $[10,12]$. Our patient was treated with azathioprine and high dose steroids, and showed significant clinical improvement.

Mortality rate in adult HLH is high, ranging from $20 \%$ to $88 \%$, mainly due to secondary infections, refractory HLH and progression of the underlying secondary causes [1].

The co-occurrence of SLE and HLH have overlapping clinical features and hence high level of suspicion is necessary for diagnosis. Prompt initiation of treatment for HLH is of extreme importance as it is a rapidly progressive life-threatening condition, even with adequate treatment.

\section{Conclusions}

SLE in males and SLE presenting as HLH is rare. HLH and SLE has overlapping clinical features and thus possess diagnostic challenges. Treatment of secondary HLH is aimed at treating the underlying cause. High-dose steroids and immunosuppressive agents are effective in SLEinduced HLH. Mortality rate of HLH is high and hence prompt initiation of treatment is of utmost importance.

\section{Additional Information Disclosures}

Human subjects: Consent was obtained by all participants in this study. Conflicts of interest: In compliance with the ICMJE uniform disclosure form, all authors declare the following:

Payment/services info: All authors have declared that no financial support was received from any organization for the submitted work. Financial relationships: All authors have declared that they have no financial relationships at present or within the previous three years with any organizations that might have an interest in the submitted work. Other relationships: All authors have declared that there are no other relationships or activities that could appear to have influenced the submitted work. 


\section{References}

1. La Rosee P, Horne A, Hines M, et al.: Recommendations for the management of hemophagocytic lymphohistiocytosis in adults. Blood. 2019, 133:2465-2477.

10.1182/blood.2018894618

2. Gupta D, Mohanty S, Thakral D, Bagga A, Wig N, Mitra DK: Unusual association of hemophagocytic lymphohistiocytosis in systemic lupus erythematosus: cases reported at tertiary care center. Am J Case Rep. 2016, 17:739-744. 10.12659/ajcr.899433

3. Petri M, Orbai AM, Alarcon GS, et al.: Derivation and validation of the systemic lupus international collaborating clinics classification criteria for systemic lupus erythematosus. Arthritis Rheum. 2012, 64:2677-2686. 10.1002/art.34473

4. Jordan MB, Allen CE, Weitzman S, Filipovich AH, McClain KL: How I treat hemophagocytic lymphohistiocytosis. Blood. 2011, 118:4041-4052. 10.1182/blood-2011-03-278127

5. Bergsten E, Horne A, Arico M, et al.: Confirmed efficacy of etoposide and dexamethasone in HLH treatment: long-term results of the cooperative HLH-2004 study. Blood. 2017, 130:27282738. 10.1182/blood-2017-06-788349

6. Trottestam H, Horne A, Arico M, et al.: Chemoimmunotherapy for hemophagocytic lymphohistiocytosis: long-term results of the HLH-94 treatment protocol. Blood. 2011, 118:4577-4584. 10.1182/blood-2011-06-356261

7. Mahlaoui N, Ouachee-Chardin M, de Saint Basile G, Neven B, Picard C, Blanche S, Fischer A: Immunotherapy of familial hemophagocytic lymphohistiocytosis with antithymocyte globulins: a single-center retrospective report of 38 patients. Pediatrics. 2007, 120:622-628. 10.1542/peds.2006-3164

8. Al-Salama ZT: Emapalumab: first global approval. Drugs. 2019, 79:99-103. 10.1007/s40265018-1046-8

9. Marsh RA, Allen CE, McClain KL, et al.: Salvage therapy of refractory hemophagocytic lymphohistiocytosis with alemtuzumab. Pediatr Blood Cancer. 2013, 60:101-109. 10.1002/pbc. 24188

10. So MW, Koo BS, Kim YJ, Kim YG, Lee CK, Yoo B: Successful rituximab treatment of refractory hemophagocytic lymphohistiocytosis and autoimmune hemolytic anemia associated with systemic lupus erythematosus. Mod Rheumatol. 2014, 24:855-857. 10.3109/14397595.2013.874740

11. Aoyama-Maeda N, Horino T, Ichii O, Terada Y: Macrophage activation syndrome associated with systemic lupus erythematosus treated successfully with the combination of steroid pulse, immunoglobulin and tacrolimus. Rom J Intern Med. 2018, 56:117-121. 10.1515/rjim2017-0043

12. Ideguchi H, Ohno S, Takase K, Hattori H, Kirino Y, Takeno M, Ishigatsubo Y: Successful treatment of refractory lupus-associated haemophagocytic lymphohistiocytosis with infliximab. Rheumatology (Oxford). 2007, 46:1621-1622. 10.1093/rheumatology/kem205 